\title{
EVALUATION OF QUAD-CONSTELLATION GNSS PRECISE POINT POSITIONING IN EGYPT
}

\author{
Emad El Manaily ${ }^{1}$, Mahmoud Abd Rabbou ${ }^{2}$, Adel El-Shazly ${ }^{3}$ and Moustafa Baraka ${ }^{4}$ \\ Ph.D. Candidates in Geomatic Engineering - Cairo University ${ }^{1}$ \\ Assistant Prof. of Surveying and Geodesy, Faculty of Engineering - Cairo University ${ }^{2}$ \\ Prof. of Surveying and Geodesy, Faculty of Engineering - Cairo University ${ }^{3}$ \\ Prof. of Surveying and Geodesy, the German University in Cairo (GUC) ${ }^{4}$
}

\begin{abstract}
:
Commonly, relative GPS positioning technique is used in Egypt for precise positioning applications. However, the requirement of a reference station is usually problematic for some applications as it limits the operational range of the system and increases the system cost and complexity On the other hand; the single point positioning is traditionally used for low accuracy applications such as land vehicle navigation with positioning accuracy up to 10 meters in some scenarios which caused navigation problems especially in downtown areas. Recently, high positioning accuracy can be obtained through Precise Point Positioning (PPP) technique in which only once GNSS receiver is used. However, the major drawback of PPP is the long convergence time to reach to the surveying grade accuracy compared to the existing relative techniques. Moreover, the PPP accuracy is significantly degraded due to shortage in satellite availability in urban areas. To overcome these limitations, the quad constellation GNSS systems namely; GPS.GLONASS, Galileo and BeiDou can be combined to increase the satellite availability and enhance the satellite geometry which in turn reduces the convergence time. In Egypt, at the moment, the signals of both Galileo and BeiDou could be logged with limited number of satellites up to four and six satellites for both Systems respectively. In this paper, we investigated the performance of the Quad-GNSS positioning in both dual- and single-frequency ionosphere free PPP modes for both high accurate and low cost navigation application, respectively. The performance of the developed PPP models will be investigated through GNSS data sets collected at three Egyptian cities namely, Cairo, Alexandria and Aswan.
\end{abstract}

Key Words: GNSS, GPS, GLONASS, Galileo, PPP

\section{INTRODUCTION}

In Egypt, although Real Time Kinematic (RTK) GPS positioning technique is used mainly in surveying applications which are required high positioning accuracy, the hardware cost due to the fact that at least two receivers are required becomes a major concern in practice. In addition, the radio or cellular communication is also necessary which is in some cases especially in downtown areas become problematic due to poor link connections. In order to reduce these operational complexities and cost, a precise point positioning (PPP) technique proposed by Zumberge et al. (1997) without needs for separate base stations and 
communication links can be used currently in Egypt replacing the existence positioning techniques. Compared to differential GPS (DGPS) and RTK system, PPP has several advantages: a PPP client is completely independent, since no base station or network of base stations is necessary. Therefore PPP can save time, resources and data volumes which have to be usually transferred between reference and rover. There is no need for simultaneous observations and no tight limit in range thanks to globally precise correction products. Thus, it is imaginable that in the near future PPP will be able to substitute not only post-processing of network solutions but also real-time differential GPS or even RTK in many applications. Nowadays, PPP is used in the agricultural industry for precision farming, disaster monitoring, in hydrography and deformation monitoring (Geng et al, 2013 and $\mathrm{Xu}$ et al, 2013). Further PPP applications are sensor positioning in seafloor mapping and marine construction as well as airborne mapping (Bisnath et. al., 2009).

The achieved accuracy of PPP depends mainly on the proper modeling of GNSS errors and bias and satellite availability. While the first order ionospheric effect is canceled out by using the un-differenced ionosphere free linear combination of GNSS code and phase measurements, IGS precise orbital and clock products is used to account for the satellite orbits and clock errors. Moreover, the tropospheric error component can be modeled sufficiently by using number of empirical models such as Saastamoinen and Hopfield models or can be eliminated by using regional tropospheric correction models such as the NOAATrop model (Gutman et. al., 2003). Other errors and bias such as the effects of ocean loading, earth tide, carrier-phase windup, sagnac, relativity, and satellite antenna phase-center variations can be rigorously modeled (Kouba et. al., 2009). On the other side, the major drawback of PPP is the long convergence time to reach to centimeter positioning accuracy due to the satellite geometry. The satellite geometry can be enhanced by adding the newly constellation satellites. The additional observations can enhance the satellite geometry and reduce the convergence time of the PPP (Rabbou et. al., 2015 and Li et. al., 2015). However, using the newly constellation satellites which uses different references systems, produces what is called inter-system biases, which can be considered as additional unknown in addition to the receiver clock bias. The positioning accuracy of a Precise Point Positioning is mainly depending on the ability to mitigate errors and biases which affect GNSS observations. These errors and biases include the satellite/receiver clock errors, satellite/receiver hardware delays, ionospheric and tropospheric delays, and multipath. Moreover, using multi-constellations satellite systems, with different references frames introduces additional errors such as time offset between systems, due to the fact that each system uses a different time frame. The GPS system uses the GPS time system (GPST), which is referenced to coordinated universal time (UTC) as maintained by the US Naval Observatory (USNO). The GLONASS time is closely related to the UTC but has a constant offset of three hours reflecting the difference between Moscow time and Greenwich time. This relation implies leap seconds for the GLONASS time. Apart from the constant offset, the difference between GLONASS time and UTC shall be within 1 millisecond arising from the keeping of the time scales by different clocks. On the other hand, the Galileo satellite system has its own time frame, namely the Galileo system time (GST), which is a continuous atomic time scale with a nominal constant offset with respect to the international atomic time (TAI). However, BeiDou Time (BDT), related to UTC through UTC (NTSC - National Time Service Center of Chinese Academy of Science). BDT offset will respect to UTC is controlled within 100 ns (modulo 1 second).

In this paper, both single and dual frequency based PPP models are assessed. A single frequency PPP model combines the observations of current GNSS constellations, including GPS, GLONASS, Galileo and Beidou. The MGEX IGS final precise products are utilized to 
account for the orbital and clock errors. The GNSS inter-system biases are treated as additional unknowns in the estimation process. The contribution of the additional GNSS observations to the single-frequency PPP is assessed through solution comparison with the traditional GPS-only counterpart. Various GNSS combinations are considered in the assessment, including GPS/GLONASS, GPS/Galileo, GPS/BeiDou and Quad-GNSS. While, a traditional dual frequency PPP observation model that uses ionosphere-free linear combinations between code observations

\section{MULTI-CONSTELLATION GNSS PPP MATHEMATICAL MODELS}

Assuming that the observations are taking simultaneously from a multi-GNSS receiver, which uses GPS time as a reference, the dual-frequency GNSS ionosphere-free observation equations can be written as (Abd Rabbou, 2015):

$$
\begin{aligned}
& P_{3 G}=\rho_{G}+c\left[d t_{r}+B_{G}^{r}\right]-c\left[d \bar{t}_{G}^{s}\right]+T_{G}+e_{G} \\
& P_{3 J}=\rho_{J}+c\left[d t_{r}+B_{G}^{r}\right]-c\left[d \bar{t}_{G}^{s}\right]+T_{J}+c\left[D I S B_{J}\right]+e_{J} \\
& \Phi_{3 G}=\rho_{G}+c\left[d t_{r}+B_{G}^{r}\right]-c\left[d \bar{t}_{G}^{s}\right]+T_{G}+\left(\bar{\lambda} \bar{N}+\Delta B^{r}+\Delta B^{s}\right)_{G}+\varepsilon_{G} \\
& \left.\Phi_{3 J}=\rho_{J}+c\left[d t_{r}+B_{G}^{r}\right]-c\left[d \bar{t}_{G}^{s}\right]+T_{J}+c\left[D I S B_{J}\right]+\overline{(\lambda} \bar{N}+\Delta B^{r}-\Delta B^{s}\right)_{J}+\varepsilon_{J}
\end{aligned}
$$

where $G$ and $J$ refer to GPS, other GNSS systems observations, respectively; DISB is the dual-frequency inter-system bias; $B^{r}, B^{S}$ are ionosphere-free differential code biases for receiver and satellites, respectively $\Delta B^{r}$ is the difference between receiver differential code and phase biases; $\Delta B^{S}$ is the difference between satellite differential code and phase biases. As can be seen from Equations (3) and (4), the un-calibrated biases such as $\Delta B^{r}$ and $\Delta B^{s}$ are lumped with the ambiguity parameters.

For single frequency PPP model, the mathematical model of the quad constellation GNSS PP can be written as (Abd Rabbou and El-Rabbany, 2015)

$$
\begin{aligned}
& P_{G}=\rho_{G}+c\left(d t_{r}+d_{G}\right)-c d \bar{t}_{G}^{s}-c\left(F_{G}^{s}\right)+T_{G}+I_{G}+e_{G} \\
& P_{J}=\rho_{J}+c\left(d t_{r}+d_{G}\right)-c d \bar{t}_{J}^{s}-c\left(F_{J}^{s}\right)+c\left[S I S B_{J}\right]+T_{J}+I_{J}+e_{J} \\
& \Phi_{G}=\rho_{G}+c\left(d t_{r}+d_{G}\right)-c d \bar{t}_{G}^{s}-c\left(F_{G}^{s}\right)+T_{G}-I_{G}+\left[\lambda \bar{N}_{G}+c\left(\delta_{G}-d_{G}\right)-c\left(\delta_{G}^{s}-d_{G}^{s}\right)\right]+\varepsilon_{G} \\
& \Phi_{J}=\rho_{J}+c\left(d t_{r}+d_{G}\right)-c d \bar{t}_{J}^{s}-c\left(F_{J}^{s}\right)+c\left[S I S B_{J}\right]+T_{J}-I_{J}+\left[\lambda \overline{N_{J}}+c\left(\delta_{J}-d_{J}\right)-c\left(\delta_{J}^{s}-d_{J}^{s}\right)\right]+\varepsilon_{J}
\end{aligned}
$$

Where $\mathrm{d} \bar{t}^{s}$ is the satellite clock error lumped with the ionosphere-free differential code bias, which can be obtained from the IGS-MGEX; $F$ is a bias term representing the combined effect of differential code bias of the satellite obtained from The IGS-MGEX archive (Abd Rabbou and El-Rabbany, 2016); SISB is single-frequency the inter-system biases. The GPS receiver hardware delay $d_{G}$ is lumped to the receiver clock error and the combined receiver clock bias is considered as a single unknown in our estimation filter.

The Extended Kalman filter is used to process the raw GNSS pseudorange and phase measurements to produce estimates state vector unknown parameters as presented in Jekeli (2001). For both single and dual-PPP models, the UNB3 tropospheric model, consisting of the Saastamoinen vertical propagation delay model and Niell mapping function, is used to account for the dry tropospheric component (Leandro et al. 2008). As The effects of ocean 
loading, Earth tide, carrier-phase windup, sagnac, relativity, and satellite antenna phasecenter variations are rigorously modeled as detailed in Kouba (2009). The estimation state vector consists of the three GNSS receiver positions mainly latitude, longitude, and altitude, receiver clock, the troposphere wet component, GPS/GLONASS, GPS/Galileo and GPS/BeiDou inter-system biases, and the float ambiguity parameters. The complete state vector for the developed model can be written as;

$$
\delta x=\left[\delta \phi, \delta \lambda, \delta h, c \bar{d} t_{r}, T_{w}, c I S B_{R}, c I S B_{E}, c I S B_{C}, A_{1}, \ldots A_{n}\right]
$$

where $\delta, \delta \lambda$ and $\delta h$ are a three-dimensional vector representing the positioning errors in latitude, longitude and altitude; $\bar{d} t_{r}$ is the GPS receiver clock bias. $\operatorname{cIS} B_{R}, \operatorname{cIS} B_{E}$ and $c I S B_{C}$ are GPS/GLONASS, GPS/Galileo and GPS/BeiDou inter-system biases, respectively in meters. $A$ is the float ambiguity in meters as described in both PPP models.

$$
\begin{aligned}
& A_{\text {dual }}=\bar{\lambda} \bar{N}+\Delta B^{r}-\Delta B^{s} \\
& A_{\text {sin gle }}=\lambda \overline{N_{J}}+c\left(\delta_{J}-d_{J}\right)-c\left(\delta_{J}^{s}-d_{J}^{s}\right)
\end{aligned}
$$

Except the ambiguity parameters, the state vector corresponding errors are stochastically defined as random walk process with spectrum densities $10^{-5} \mathrm{~m}^{2} / \mathrm{sec}$ for positioning parameters, $10^{10} \mathrm{~m}^{2} / \mathrm{sec}$ for the receiver clock bias, $10^{-8} \mathrm{~m}^{2} / \mathrm{sec}$ for the troposphere wet component and $10^{-10} \mathrm{~m}^{2} / \mathrm{sec}$ for GNSS inter-system biases for both dual and single modes. The ambiguity parameters are defined stochastically as constant values. The GNSS observations are assumed to be uncorrelated and followed the Gaussian distribution with zero mean. As a result, the variance-covariance matrix takes the form of a diagonal matrix with a 100 times ratio between the GNSS code and phase observation precision. The GPS and GLONASS code and phase observation precision is set to be 0.1 and $0.001 \mathrm{~m}$, respectively. According to Steigenberger et al, (2015), the clock and orbital products for Galileo and BeiDou are less accurate compared with GPS clock and orbital products. As a result, the Galileo and BeiDou code and phase observations are weighted by $1 / 4$ with a precision taken as 0.2 and $0.002 \mathrm{~m}$, respectively

\section{GNSS STATIC DATA PROCESS}

The datasets collected at three selected stations in Egypt at different latitudes to cover the country namely: Cairo, Alexandria and Aswan on two consecutive days, i.e. July 14-15, 2015, are used for numerical analysis as appeared in figure 1.

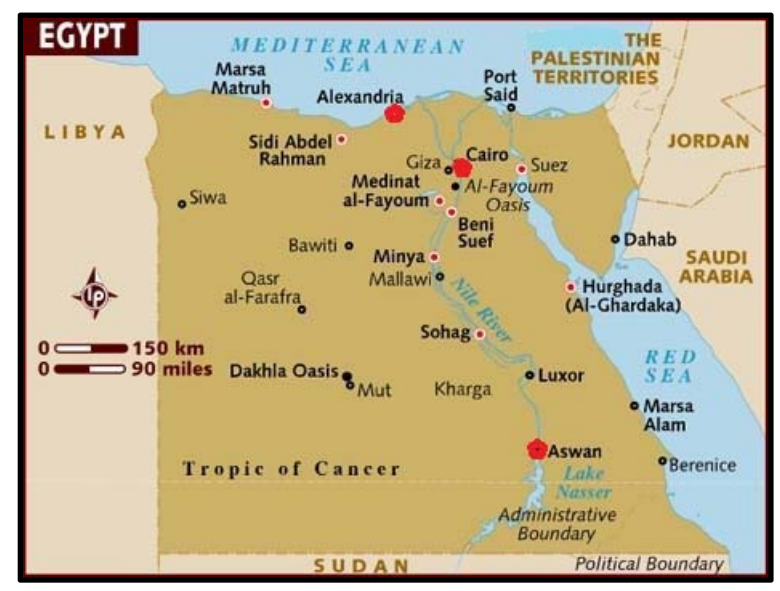

Fig.1. The three selected GNSS stations namely at Cairo, Alexandria and Aswan

Figure 2 shows the GNSS availability and GDOP for the quad constellation at Cairo for the first day of our numerical analysis. It is obvious that maximum of four satellites of Galileo 
can be tracked while six to seven satellites of BeiDou can be tracked at Cairo. It can also be seen that, except Galileo, there are sufficient number of both GLONASS and BeiDou all the day. However, there are certain GDOP jumps for both GPS and GLONASS which probably are due to the low elevation angles of part of the satellites which significantly worsen the GDOP. These jumps are hardly appeared in GNSS GDOP due to the addition of GNSS satellites which significantly enhance the GDOP in comparison with the GDOP of the GPS only. However, this contribution attributes mainly from the additional GLONASS observations while the GDOP for the combined GPS/Galileo is marginally enhanced due to the limited number of Galileo satellites available.
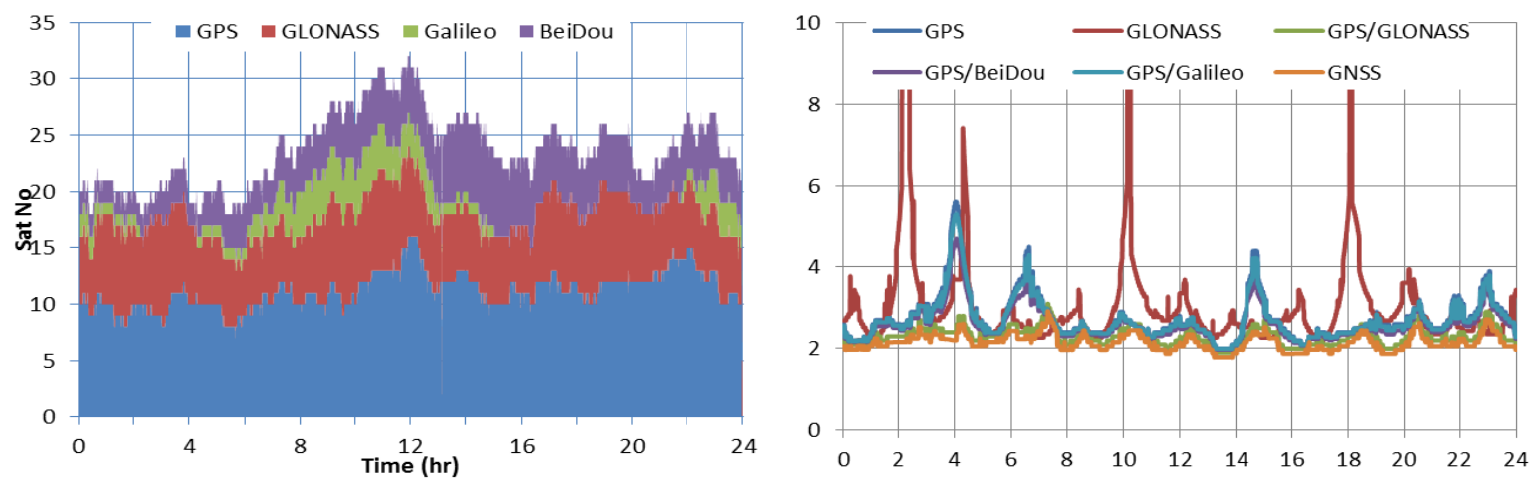

Fig. 2. The Quad- Constellation GNSS satellites availability and GDOP for Cairo at July 14, 2015.

\section{GNSS POSITIONING RESULTS AND ANALYSIS}

The observations from GPS L1/L2, GLONASS G1/G2, Galileo E1/E5a and BeiDou B1/B2 signals are adopted for dual-frequency PPP model while only the signals on L1 is used for the quad constellation for single frequency PPP model. The BeiDou and Galileo antenna offsets recommended by the MGEX project are used to correct the PCOs of BeiDou and Galileo satellites (Rizos et al 2013). To evaluate the performance of different GNSS combinations, namely GPS only, GLONASS only, GPS/GLONASS, GPS/Galileo, GPS/BieDou and GPS/GLONASS/Galielo/BeiDou (GNSS) three-hour position solutions are analyzed to represent the PPP performance in a short observation time. For the two days datasets, four data sessions are selected for each day with minimum two satellites from each Galileo and BeiDou systems, are available. Each session is processed separately so that a total of 24 sets of results are obtained for each GNSS combination to derive a statistical estimate on the positioning accuracy and convergence time.

Figure 3 shows the positioning error results of dual-frequency PPP model for station Alexandria for one hour of processing data as an example. As can be seen that compared with the existing GPS PPP positioning solution, the additional Galileo observations have a marginal effect on the positioning accuracy and convergence time. The main contribution is attributed to the additional GLONASS observations due to the sufficient GLONASS observations available. Additionally, compared with the combined GPS/GLONASS PPP, the quad constellations combined GNSS PPP present comparable positioning accuracy. The insignificant effect is due to the sufficient number of satellite observations originally available from both GPS and GLONASS. In addition, the GLONASS only solutions present worsen positioning accuracy compared with the GPS only solution with respect to the convergence time. 

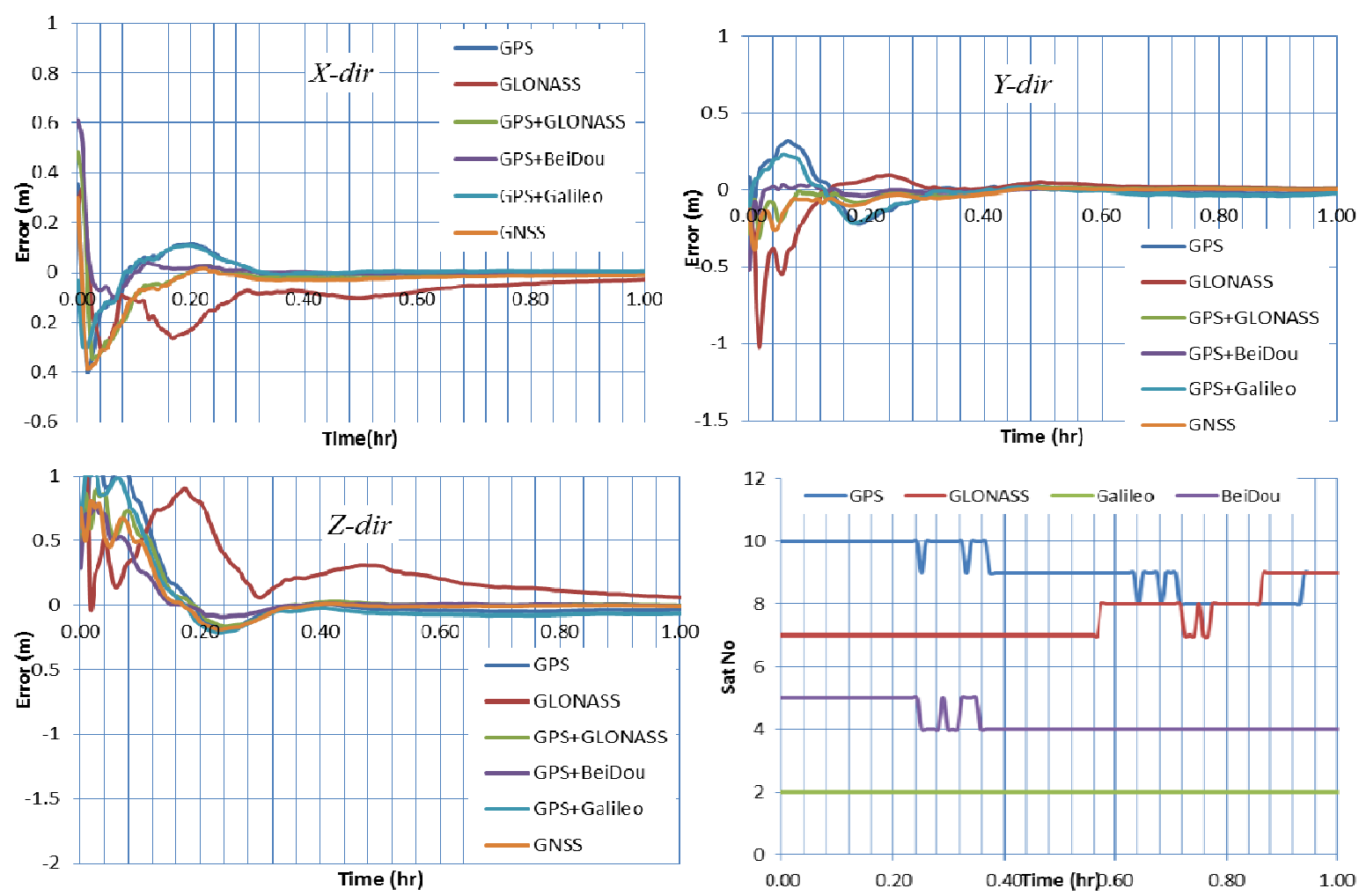

Fig. 3. Dual-frequency ionosphere-free PPP model positioning results with satellite avaialbilty

Table 1 shows the root mean square error RMSE from the results obtained from all the data sets processed for different processing times. Due to the limited number of Galileo satellites, the contribution of Galileo observations can be considered insignificant on enhancing the GPS PPP positioning accuracy. In most cases, both GPS and GPS/Galileo PPP present same accuracy level which attribute to the limited number of Galileo satellites. The additional BeiDou observations enhanced the positioning accuracy by $12 \mathrm{~cm}$ compared with GPS only positioning accuracy after 5 mins of processing data while after 30 mins only $2 \mathrm{~cm}$ accuracy improvement can be detected. The GLONASS only solutions present less accurate positioning solution compared with the GPS only. Compared with GPS PPP, the GNSS PPP enhanced the positioning accuracy by $30 \mathrm{~cm}$ after 5 mins while present comparable positioning accuracy at 120 mins of processing data. However, the positioning improvements are mainly attributed to the additional GLONASS observations. This can be easily noticed when the GPS/GLONASS PPP and GNSS PPP positioning accuracy are compared, the additional Galileo and BeiDou observations has a marginal effect when they added to the GPS and GLONASS observations

Table 1. The 3D positioning accuracy for different GNSS PPP combinations after different processing times for dual-frequency PPP model.

\begin{tabular}{ccccccc}
\hline Time & \multicolumn{5}{c}{ 3D positioning accuracy $(\mathrm{m})$} \\
\cline { 2 - 7 }$(\min )$ & GPS & GLONASS & GPS/GLONASS & GPS/BeiDou & GPS/Galileo & GNSS \\
5 & 0.99 & 1.09 & 0.64 & 0.87 & 0.92 & 0.68 \\
10 & 0.22 & 0.92 & 0.10 & 0.18 & 0.21 & 0.11 \\
30 & 0.04 & 0.32 & 0.01 & 0.02 & 0.02 & 0.01 \\
60 & 0.04 & 0.07 & 0.01 & 0.01 & 0.05 & 0.01 \\
120 & 0.02 & 0.02 & 0.01 & 0.01 & 0.06 & 0.02 \\
\hline
\end{tabular}


Table 2 shows the comparison between the mean convergence times for the different GNSS PPP combinations. The convergence times clarified in Table 2 are computed using the average of the convergence times in the three positioning directions, namely, $\mathrm{X}, \mathrm{Y}$ and $\mathrm{Z}$, respectively, for each data sets. It can be noticed that the additional GLONASS observations reduces the positioning convergence by 7 minutes compared with GPS PPP which represent $38 \%$ in convergence time improvement. While the additional BeiDou and Galileo observations fail to reduce the convergence time when they added to the GPS/GLONASS PPP. However, compared with the GPS PPP, the additional BeiDou observations reduces the convergence time by three minutes which represent $16 \%$ in convergence time improvement. As seen in Table 3, Both GPS and GPS/Galileo present identical average convergence time due to the limited number of Galileo satellites.

Table 2. The convergence time for different GNSS combinations using dual frequency PPP

\begin{tabular}{cc}
\multicolumn{2}{c}{ model } \\
\hline Poisoning & $\mathrm{CT}(\mathrm{min})$ \\
Combination & \\
\hline GPS & 19.5 \\
GLONASS & 34.5 \\
GPS/GLONASS & 12 \\
GPS/BeiDou & 17 \\
GPS/Galileo & 19 \\
GNSS & 12 \\
\hline
\end{tabular}

For single frequency PPP model, Figure 4 shows the GNSS satellite availability and positioning errors with time for the various GNSS constellation combinations at stations Aswan, respectively, It can be seen that the major contribution to the Single frequency PPP solution enhancement is due to the additional GLONASS observations. This is due to the good availability of GLONASS compared with the other constellation, which significantly affects the overall satellite geometry. On the other hand, because of their limited number of visible satellites, the addition of Galileo and BeiDou systems has a marginal effect on the positioning accuracy, in comparison with the GPS/GLONASS PPP Positioning accuracy. 

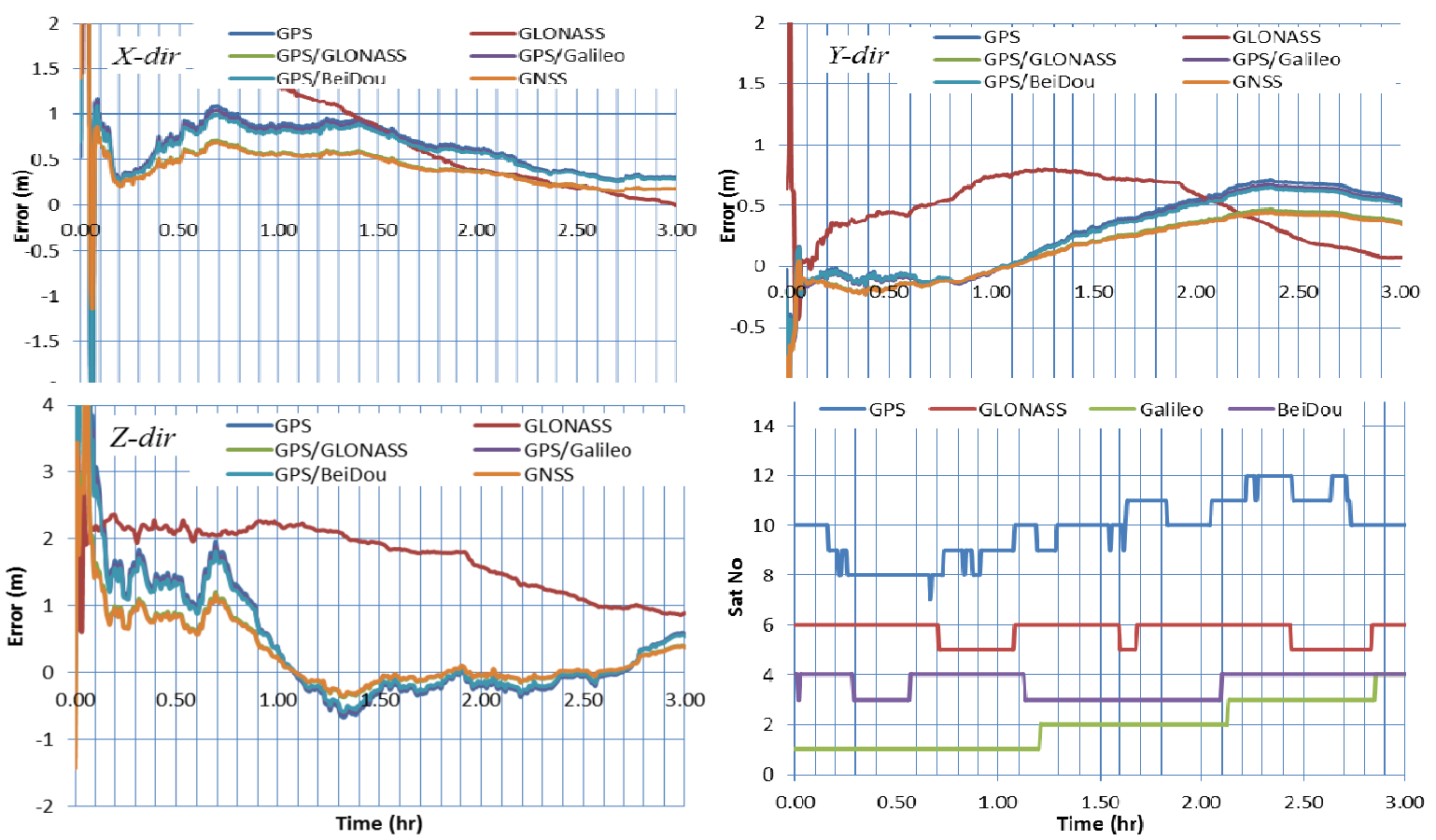

Fig. 4. The single frequency positioning accuracy and GNSS satellite availability

Table 3 shows the 3D positionig RMSE for the different GNSS combination in single frequency mode for different processing times. It can be seen the the additional GLONASS observation enhanced the positioning accuracy by $60 \mathrm{~cm}$ after 5 mins while $10 \mathrm{~cm}$ positioning accuracy improverement can be obtained after 2 hours of data processing. In addition, the positonig accuracy can be enhanced by $5 \mathrm{~cm}$ when the BeiDou observation are added after two hours of processing data. In contrast, the Galileo observations can be considered marginal when combined with the GPS observations.

Table 3. The 3D positioning accuracy for different GNSS PPP combinations after different processing times for Single-frequency PPP model.

\begin{tabular}{ccccccc}
\hline Time & \multicolumn{5}{c}{ 3D positioning accuracy $(\mathrm{m})$} \\
\cline { 2 - 7 }$(\min )$ & GPS & GLONASS & GPS/GLONASS & GPS/BeiDou & GPS/Galileo & GNSS \\
5 & 3.59 & 3.99 & 2.80 & 3.40 & 3.49 & 2.72 \\
30 & 1.28 & 3.46 & 1.08 & 1.17 & 1.75 & 0.99 \\
60 & 0.33 & 1.19 & 0.23 & 0.28 & 0.38 & 0.20 \\
120 & 0.32 & 0.42 & 0.22 & 0.28 & 0.31 & 0.21 \\
240 & 0.28 & 0.26 & 0.23 & 0.23 & 0.24 & 0.22 \\
\hline
\end{tabular}

\section{CONCLUSION}

In this paper, we investigated the performance of both dual and Single-frequency quad constellation PPP which combines the observations of current GNSS constellations, including GPS, GLONASS, Galileo and Beidou for precise and low cost positioning applications, respectively in Egypt. The IGS-MGEX final precise products were used to account for the orbital and clock errors, respectively, while the final IGS GIM model was used to account for the ionospheric delay for the single-frequency PPP model. Different GNSS PPP combinations were assessed namely, GPS only, GLONASS only, GPS/GLONASS, GPS/BeiDou GP/Galielo and quad-constellation GNSS PPP. For dual frequency PPP model, the results indicate that the convergence time for the combined GNSS PPP was enhanced by 35\% 
compared with the standard GPS PPP convergence time. Moreover, the additional BeiDou observations enhanced the positioning accuracy by $5 \mathrm{~cm}$, compared with GPS only positioning accuracy while the additional Galileo satellite was found to be insignificant when its observations were added to the GPS observations. Compared with GPS PPP, the GNSS PPP enhanced the positioning accuracy by $8 \mathrm{~cm}$. However, the positioning improvements are mainly attributed to the additional GLONASS observations. For single-frequency PPP model, the contribution of the additional GNSS observations to the PPP solution was assessed through comparison with the traditional GPS-only counterpart. It was shown that the contribution of the additional GLONASS observations is significant, while the contribution of both Galileo and BeiDou can be consider marginal due to their limited satellite availability.

\section{REFERENCES}

Abd Rabbou, M. and El-Rabbany, A. (2015), "Precise Point Positioning Using MultiConstellation GNSS Observations for Kinematic Applications". Journal of Applied Geodesy, 9, 15-26. http://dx.doi.org/10.1515/jag-2014-0021

Abd Rabbou M, El-Rabbany A (2016), "Performance analysis of precise point positioning using multi-constellation GNSS: GPS, GLONASS, Galileo and BeiDou”. Surveying review, May 2016

Bos, M.S. and Scherneck, H.-G. (2011), “Ocean Tide Loading Provider". http:/holt.oso.chalmers.se/loading/

Bisnath, S. B. and Gao, Y. (2009), "Innovation: Precise Point Positioning - A Powerful Technique with a promising future”. GPS World, April 2009

Gutman, S., Fuller-Rowell, T. and Robinson, D. (2003), "Using NOAA Atmospheric Models to Improve Ionospheric and Tropospheric Corrections". Proceedings of the US Coast Guard DGPS Symposium, Portsmouth, 17-19 June 2003.

Jekeli, C. (2001), "Inertial Navigation Systems with Geodetic Applications". Walter de Gruyter, Berlin. http://dx.doi.org/10.1515/9783110800234

Kouba, J. (2009), “A Guide to Using International GNSS Service (IGS) Products”. http://igscb.jpl.nasa.gov/igscb/resource/pubs/UsingIGSProductsVer21.pdf

Leandro, R.F., Langley, R.B. and Santos, M.C. (2008), "UNB3m_pack: A Neutral Atmosphere Delay Package for Radiometric Space Techniques". GPS Solutions, 12, 6570. http://dx.doi.org/10.1007/s10291-007-0077-5

Li, X., Zhang, X., Ren, X., Fritsche, M., Wickert, J. and Schuh, H. (2015), "Precise positioning with current multi-constellation Global Navigation Satellite Systems: GPS, GLONASS, Galileo and BeiDou". Scientific reports, 5. 
Steigenberger P, Hugentobler U, Loyer S, Perosanz F, Prange L, Dach R, Uhlemann M, Gendt G, Montenbruck O (2015), "Galileo orbit and clock quality of the IGS Multi-GNSS Experiment”. Advances Space Research, volume 55, issue 1:269-281.

Zumberge, J.F., Heflin, M.B., Jefferson, D.C., Watkins, M.M. and Webb, F.H. (1997), "Precise point positioning for the efficient and robust analysis of GPS data from large networks". Journal of Geophysical Research, Vol. 102, 5005-5017.

Received: 2016-12-13,

Reviewed: 2017-01-17, by P. Václavovic, and 2017-02-06, by Jianghui Geng,

Accepted: 2017-03-13. 